\title{
Metabolic Surgery: Paradigm Shift in Metabolic Syndrome/Diabetes Therapy
}

\author{
Annika Rühle Adrian T. Billeter Beat P. Müller-Stich \\ Department of General, Visceral, and Transplantation Surgery, University of Heidelberg, Heidelberg, Germany
}

\section{Keywords}

Metabolic surgery · Type 2 diabetes · Obesity · Metabolic dysfunction fatty liver disease $\cdot$ Cardiovascular risk

\begin{abstract}
Background: Obesity and metabolic disorders as type 2 diabetes (T2D), nonalcoholic fatty liver disease (NAFLD) or better called metabolic dysfunction fatty liver disease (MAFLD), arterial hypertension (AHT), and obstructive sleep apnea syndrome (OSAS) show a rising prevalence. The increased cardiovascular risk is one of the main causes for death of obese, metabolic ill patients. Sustainable and efficient therapeutic options are needed. Summary: Metabolic surgery not only permits a substantial and lasting weight loss but also ameliorates metabolic co-morbidities and reduces cardiovascular risk and mortality of obese patients. Most existing data focused on T2D, but evidence for other metabolic comorbidities such as NAFLD, AHT, and OSAS increase constantly. After metabolic surgery, glycemic control of diabetic patients is superior compared to conservative treatment. Also, diabetes related micro- and macrovascular complications are reduced after surgery, and the median life expectancy is over 9 years longer. In patients with MAFLD, metabolic surgery leads to reduction of steatosis and fibrosis while the risk to develop a hepatocellular carcinoma is reduced significantly. Patients with OSAS have an improved lung function and continuous pressure airway treatment during the night is unnecessary in many patients. Patients with AHT need significantly less or even no antihypertensive medication after surgery and the hazard ratio of death is reduced by $49.2 \%$. Therefore, the focus in treating obese and metabolic ill patients is no longer on pure weight loss but on improvement of co-morbidities and reduction of mortality.
\end{abstract}

This is reflected by the updated S3-guidelines of 2018 that provide nationally established consistent guidelines with clear indications for metabolic surgery no longer focusing on body mass index (BMI) only. This article aims to give an overview over the existing literature concerning surgical treatment options for metabolic syndrome. Key Messages: Metabolic co-morbidities impact life-quality and life expectancy of obese patients. Metabolic surgery offers the chance to treat those metabolic co-morbidities independently of the preoperative BMI and should be considered early as a treatment option for obese patients.

(c) 2022 S. Karger AG, Basel

\section{Introduction}

Due to the rising prevalence of metabolic disorders such as obesity, type 2 diabetes (T2D), nonalcoholic fatty liver disease (NAFLD) or better metabolic dysfunction associated fatty liver disease (MAFLD), arterial hypertension (AHT), and obstructive sleep apnea syndrome (OSAS), efficient and sustainable therapeutic options are needed. Over the last decades, the surgical therapy proved its effectiveness in various trials not only concerning a substantial and lasting weight loss but also by reducing cardiovascular risk and mortality $[1,2]$. Observations also showed that the risk of developing metabolic co-morbidities such as T2D was lower after bariatric surgery [3] while existing T2D or MAFLD often resolved [4-6]. Interestingly, these effects were not strictly associated with the postoperative weight loss and could be detected in patients with lower body mass indices (BMIs) as well [7]. Therefore, metabolic surgery evolved, with the treatment focus not on weight loss but on treatment of metabolic 


\section{Primary indication}

- $\mathrm{BMI}>50 \mathrm{~kg} / \mathrm{m}^{2}$

- $\mathrm{BMl}>40 \mathrm{~kg} / \mathrm{m}^{2}$

$+\mathrm{T} 2 \mathrm{D}$

- Intedsiciplinary consensus on hopeless conservative treatment or severity of co-morbidities

\section{Secondary indication}

- $\mathrm{BMl}>40 \mathrm{~kg} / \mathrm{m} 2$

+ failure of conservative therapy

- $\mathrm{BMl}>35 \mathrm{~kg} / \mathrm{m} 2$

+ metabolic co-morbidity*

+ failure of conservative therapy

- T2D

+ BMI $35-40 \mathrm{~kg} / \mathrm{m} 2$

+ failure of therapy goals ( $\mathrm{HbA} 1 \mathrm{c})$

- T2D

+ BMI $30-35 \mathrm{~kg} / \mathrm{m} 2$

+ bad glycemic control

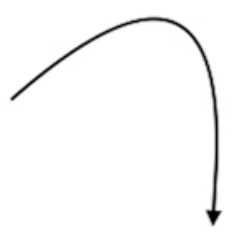

Documentation (Diary)

- Excercise

- Weight

- Nutrition

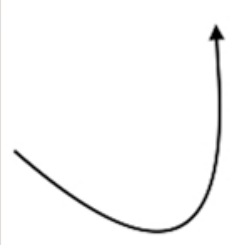

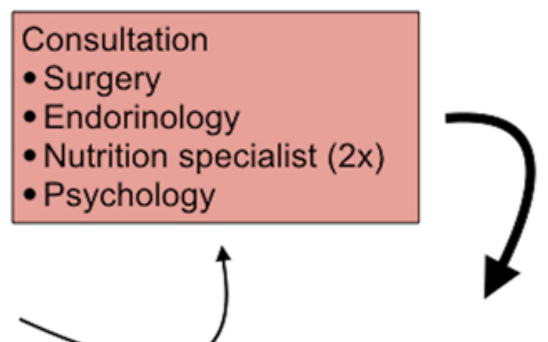

Metabolic surgery

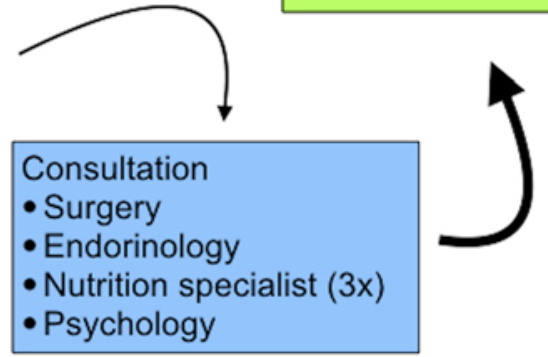

* Type 2 diabetes (T2D), coronary heart disease, cardiac insufficiency, dyslipidemia, arterial hypertension, obstructive sleep apnea (OSAS), Pickwick-Snydrom, metabolic dysfunction associated fatty liver disease (MAFLD), non-alcoholic steatohepatitis (NASH), pseudotumor cerebri, gastroesophageal reflux disease, asthma, chronic venous insufficiency, urine incontinence, immobilizing joint disease, restraint fertility or polycystic ovarial syndrome

Fig. 1. Indication for metabolic surgery according to the current S3-guidelines.

co-morbidities such as AHT, MAFLD, OSAS, and not at least T2D [8]. Recent studies further confirm that metabolic surgery exerts additional beneficial effects on cardiovascular events that go beyond weight loss [9]. As a result of these developments, the S3-guidelines for treatment of obesity and metabolic co-morbidities were updated in 2018 [10]. This was a turning point in the treatment of obese patients with metabolic disorders since nationally established consistent guidelines defined clear indications for metabolic surgery that no longer focus on BMI only, but metabolic co-morbidities. Most existing literature focuses on $\mathrm{T} 2 \mathrm{D}$, but there is growing evidence for other metabolic co-morbidities such as MAFLD, OSAS, and AHT being ameliorated by metabolic surgery. So far, existing data do not allow to indicate metabolic surgery only based on metabolic co-morbidities and completely independent of patients BMI. More randomized-controlled trials are needed to further validate existing data. This article aims to give an overview on existing literature. Figure 1 gives a brief overview on indication for surgery according to the current S3-guidelines. Table 1 gives an overview of known benefits according to existing literature that will be described below.

\section{Type 2 Diabetes}

Excessive consume of sugar leads to higher blood insulin levels. Supported by overweight or obesity, a peripheral insulin resistance emerges. With the relative lack, insulin production in the pancreas increases until finally exhaustion results in an absolute lack of insulin. Subsequently, micro- and macrovascular complications as damages to kidneys, eyes, nerves, and vessels may appear. Most common cause of death in diabetic patients remains cardiovascular events despite modern medical treatment [11]. Results of the Swedish Obese Subjects study from 2008 to 2013 showed that metabolic surgery significantly reduces cardiovascular risk and mortality $[12,13]$.

In 2021, the 10-year follow-up data of a randomizedcontrolled trial comparing medical therapy and metabolic surgery for patients with T2D were published [14]. These data confirm the existing literature regarding the short-term effects of metabolic surgery on glycemic control and prove the sustainability over 10 years. $37.5 \%$ of surgical treated patients versus 5.5\% medically treated patients maintained diabetes remission throughout the 
Table 1. Indication and known benefits of metabolic surgery

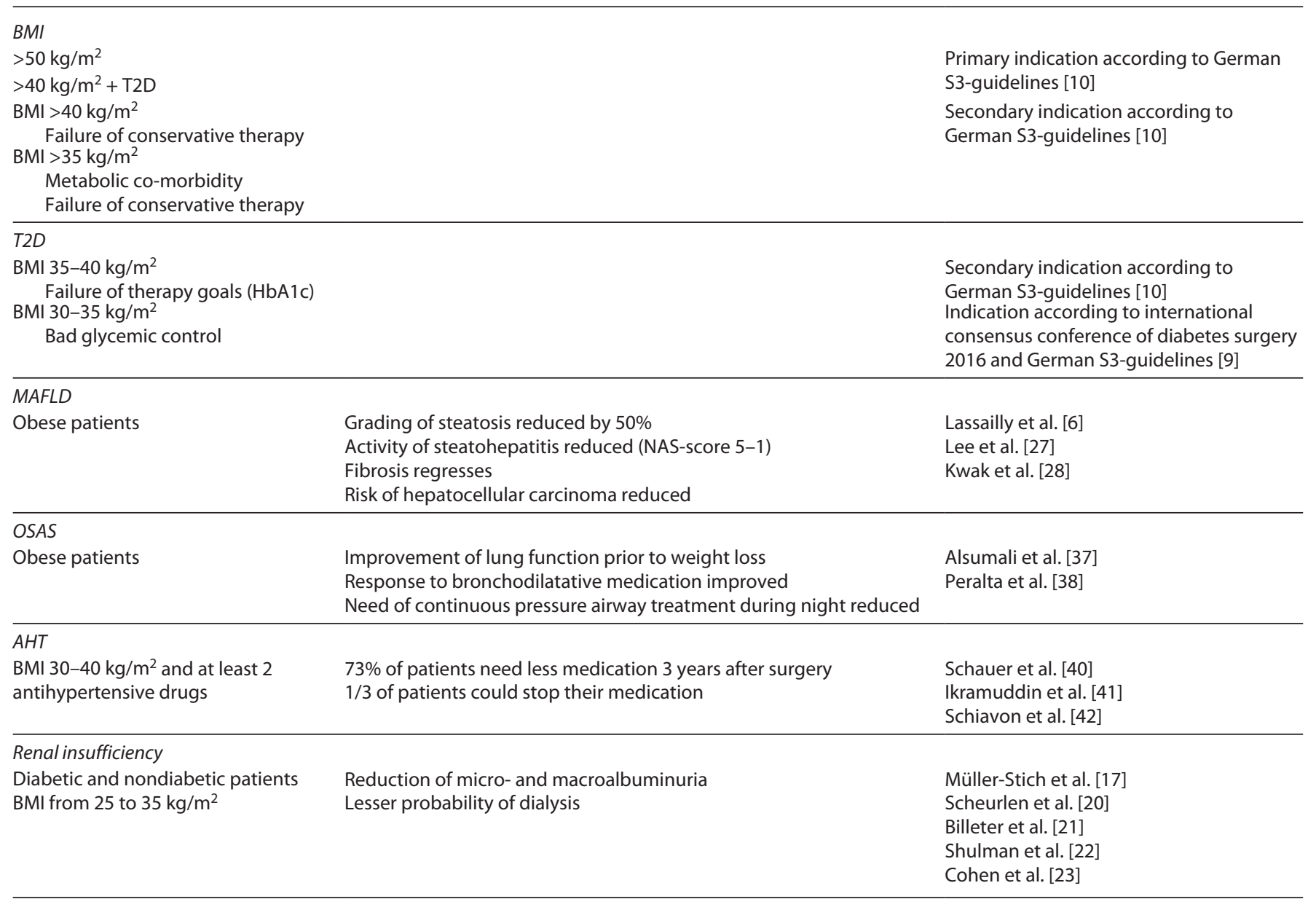

10-year follow-up. Operated patients also had fewer diabetes-related complications. Duration of T2D remission and relapse remains much debated. Due to the lack of long-term data $>20$ years, the exact number is unclear. However, the current data with 5-10-year follow-up show relapse rates of $30-70 \%$. While there is a high rate of relapse of T2D even after metabolic surgery, there is some evidence that relapse should not be considered a failure since the cardiometabolic benefits remained [15]. A major fact, that is often forgotten, is that early surgery also results in much longer T2D remission rates than in patients with long-standing, insulin-treated T2D. It is clearly established that early metabolic surgery would provide even better and stronger metabolic and life prolonging effects as shown by a recent meta-analysis from Syn et al. [16]. This study clearly demonstrated the longterm benefit of metabolic surgery. Metabolic surgery was associated with a $49.2 \%$ reduced hazard rate of death at every time point of 30 years of follow-up compared to medical therapy and the median life expectancy of diabetic patients was 9.3 years longer after metabolic surgery.
Furthermore, micro- and macrovascular complications of T2D are also ameliorated after metabolic surgery: over $50 \%$ of patients with T2D and symptomatic polyneuropathy are 6 months postoperatively completely symptom-free [17]. Meta-analyses showed that the risk to suffer from microvascular complications of diabetic patients with a BMI $\geq 27 \mathrm{~kg} / \mathrm{m}^{2}$ and above is reduced by twothirds [18]. The overall-mortality is reduced by $60 \%$; the cardiovascular mortality is reduced by $80 \%$ in diabetic patients with $B M I \geq 27 \mathrm{~kg} / \mathrm{m}^{2}[19,20]$.

Also renal function improves after metabolic surgery [21]. Interestingly, improvement already occurs in patients with a BMI from 25 to $35 \mathrm{~kg} / \mathrm{m}^{2}$ [20], and microalbuminuria is reduced and creatinine- and cystatine Cclearance is improved. The Swedish Obese Subject-study [22] as well as Cohen et al. [23] confirmed these results and furthermore found decreased macroalbuminuria. Surgical treated patients also were less likely to need dialysis than conservative treated patients. Interestingly, this could be shown in both diabetic as well as nondiabetic patients. 


\section{Metabolic Dysfunction Associated Fatty Liver Disease}

Insulin resistance leads to lipolysis in muscle and fatty tissue and DeNovo-lipogenesis in the liver. The excessive supply of fatty acids causes an overload of the liver metabolism and over time results in liver damage. Hepatic steatosis (NAFLD) can lead to steatohepatitis (NASH), liver cirrhosis, and hepatocellular carcinoma (HCC). The risk to develop a HCC is even higher in patients with T2D and can already occur before signs of cirrhosis appear [4]. Also, nonobese patients with metabolic co-morbidities can develop a then called "lean-NASH" with similarly increased risk for development of cirrhosis and HCC [24]. Recently, the term of the MAFLD was proposed for the changes in the liver associated with metabolic diseases instead the widely used terms of NAFLD and NASH [25].

Metabolic surgery effectively ameliorates NAFLD/ MAFLD/NASH: not only steatosis is reduced ( $10 \%$ vs. $60 \%$ ), activity of steatohepatitis is reduced (NAS-Score 1 vs. 5) and fibrosis regresses but also the risk of developing a HCC is reduced significantly [6,26-29]. Most recent data from Rustgi et al. [30] even detected a significant reduction of risk for development of any cancer by $18 \%$ and obesityrelated cancer by $25 \%$ in patients with NAFLD and severe obesity after bariatric surgery versus conservative treatment. Furthermore, the risk to develop a liver cirrhosis is significantly reduced after metabolic surgery in patients with NAFLD [29]. Change of diet and weight loss, independent of the BMI, leads to an improvement of MAFLD [31]. So far, there are no data for metabolic surgery on patients with MAFLD and BMI $<35 \mathrm{~kg} / \mathrm{m}^{2}$ and comparative trials of medical and surgical therapy are missing. Therefore, currently MAFLD/NAFLD/NASH is not a primary indication for metabolic surgery, but hopefully will be considered during the next update of the guidelines.

\section{Obstructive Sleep Apnea}

A large portion of overweight patients suffer from OSAS and half of the OSAS patients are overweight. Obviously, there is a mechanical element in the pathogenesis: with an accumulation of fatty tissue in throat and thoracic area the airway is narrowed, the vital capacity is reduced and therefore the breathing frequency is elevated [32]. The metabolic element is caused on the one hand by oxidative stress due to apnea episodes and by inflammatory processes mediated by adipocytokines from adipose tissue on the other hand $[33,34]$. We know, that as well as the duration of obesity ( $<5$ vs. $>15$ years) and the increase in weight has a negative effect on lung function [35]. It is also believed that OSAS itself might be a factor of additional weight gain [36]. However, the effect of
CPAP-therapy in patients with OSAS on weight is conflicting [32].

Weight loss contrariwise improves lung function and the need for continuous pressure airway treatment during the night is no longer necessary in many patients after metabolic surgery $[37,38]$. Obviously, due to the increased and ongoing weight loss after metabolic surgery compared to conservative treatment and the abovementioned pathogenesis, patients have a significantly improved lung function after surgical treatment. Interestingly, improvement in oscillometry already begins before a relevant weight loss has occurred and response to bronchodilatative medication is improved, therefore metabolic changes after metabolic surgery are believed to be causative [39]. Further studies are needed to influence existing guidelines, since OSAS itself is currently no indication for metabolic surgery as of yet.

\section{Arterial Hypertension}

AHT is one of the main risks for cardiovascular events and often occurs in obese patients. Medical therapy offers many options. As a side effect of metabolic surgery, it can be observed that postoperatively patients usually need less medical treatment for an AHT $[40,41]$. A recent randomized-trial by Schiavon et al. [42] in patients with BMI $30-40 \mathrm{~kg} / \mathrm{m}^{2}$ and at least two antihypertensive drugs, demonstrated that $73 \%$ of patients needed significantly less medication 3 years after metabolic surgery whereas only $11 \%$ of the conservative treatment group achieved this goal. Roughly, one-third of the patients were even able to stop their antihypertensive medication at all while only $0-2 \%$ accomplished that in the conservative group [42]. Therefore, AHT treated with a least two antihypertensive drugs in patients with a BMI $30-40 \mathrm{~kg} / \mathrm{m}^{2}$, can now be considered an indication for a metabolic operations. Concerning long-term data, the very recent metaanalysis of Syn et al. [16] showed in the overall survival a reduction in the hazard rate of death of $49.2 \%$ with a 6.1 years longer median life expectancy after metabolic surgery compared to conservative treatment. These data not only concerned on cardiovascular events but on overall survival. Since cardiovascular events display the main cause for mortality in obese patients, these data can be considered as representative for the reduction of cardiovascular risks and mortality after metabolic surgery.

\section{Surgical Procedures and Preoperative Evaluations}

Choosing the "right" operation should be an individualized process: on the one hand are patients' factors such as co-morbidities (e.g., gastroesophageal reflux or ulcer- 
ations as relative contraindication for a sleeve gastrectomy), profession and age, while technical aspects (e.g., extreme BMI with high amount of visceral fat as relative contraindication for roux-y gastric bypass [RYGB] due to tension on the meso) should be considered as well. Only one example is T2D as co-morbidities that should play a role in the decision-making: Aminian et al. [43] published an individualized metabolic surgery score that helps choosing the right operation, since in some patients a RYGB is superior compared to a sleeve gastrectomy concerning postoperative diabetes remission.

Importantly, patients' wishes and experiences need to be considered. However, patients' perception should be evaluated and unrealistic expectations regarding weight loss and improvement of co-morbidities need to be discussed prior to surgery to secure patients' therapy adherence. Therefore, a multimodal medical attendance, an interdisciplinary cooperation of endocrinologist, psychologist, nutritional specialist and surgeon, is advisable.

Surgical evaluations aim to reduce the perioperative risk to the absolute minimum for an elective procedure. A profound anamnesis helps to detect risk factors and point towards need for further medical evaluation. A preoperative gastroscopy is helpful to detect ulcerations and treat a Helicobacter pylori infection. Eradication lessens the risk of postoperative ulcerations.

Endocrinological evaluations aim to detect metabolic co-morbidities and to identify the underlying diseases that may cause or aggravate obesity (e.g., hypothyreosis). Postoperatively, changes in the parathyroid function (i.e., hyperparathyroidism) needs to be detected and treated. Adaption of medical treatment of co-morbidities either prior to surgery to reduce the perioperative risk or in the course after surgery is another duty of the endocrinologist.

Nutritional specialists are elementary for preoperative preparation of the patient, to assure a smooth transition to the postoperatively required change in diet. Ideally, patients already get used to smaller portions in advance. Also, a balanced nutrition with sufficient amount of protein intake needs to be ensured and deficits in vitamin supply settled.

Psychological or psychosomatic evaluation is the last column of the multimodal preoperative evaluation: obe- sity is often associated with eating disorders, substance abuse, or depression [44]. Psychological co-morbidities are not a contraindication for metabolic surgery but should be detected and treated. Patients with good therapy adherence can safely undergo metabolic surgery. Lastly, a good compliance for lifelong supplementation of vitamins and calcium as well as follow-up visits should be ensured.

Regarding the choice between the existing procedures, few studies directly compare different metabolic operations. Most trials used RYGB, the typical metabolic operation, as comparison to medical therapy. However, randomized-controlled trials in obese patients with the primary endpoint on weight loss showed comparable results of RYGB, sleeve gastrectomy, or the omega loop gastric bypass [45-47]. Improvement of metabolic co-morbidities, as secondary endpoints in these trials, was not found to be different between procedures. Further evaluations focusing on metabolic disease-control as primary endpoint are necessary.

\section{Summary}

Metabolic surgery offers the chance to treat metabolic co-morbidities, which impact life-quality and life expectancy of obese patients, independently of preoperative BMI, and should be considered early as treatment option.

\section{Conflict of Interest Statement}

The authors declare that there is no conflict of interests.

\section{Funding Sources}

There is no funding.

\section{Author Contributions}

A.R., A.T.B., and B.P.M.-S. put together the article content. The manuscript was drafted by A.R. and A.T.B. All authors have read and approved the final manuscript.

\section{References}

1 Cohen R. Revisional bariatric surgery and choosing the first procedure: twenty-six years of follow-up in the SOS study. JAMA Surg. 2019;154:326-7.

2 Wiggins T, Guidozzi N, Welbourn R, Ahmed AR, Markar SR. Association of bariatric surgery with all-cause mortality and incidence of obesity-related disease at a population level: a systematic review and meta-analysis. PLoS Med. 2020;17:e1003206.
3 Thereaux J, Lesuffleur T, Czernichow S, Basdevant A, Msika S, Nocca D, et al. Long-term adverse events after sleeve gastrectomy or gastric bypass: a 7-year nationwide, observational, population-based, cohort study. Lancet Diabetes Endocrinol. 2019;7:786-95.

4 Roeb E, Steffen HM, Bantel H, Baumann U, Canbay A, Demir M, et al. S2k Leitlinie Nichtalkoholische Fettlebererkrankungen. Z Gastroenterol. 2015;53:668-723.
5 Degasperi E, Colombo M. Distinctive features of hepatocellular carcinoma in non-alcoholic fatty liver disease. Lancet Gastroenterol Hepatol. 2016;1:156-64.

6 Lassailly G, Caiazzo R, Buob D, Pigeyre M, Verkindt H, Labreuche J, et al. Bariatric surgery provides long-term resolution of nonalcoholic steatohepatitis and regression of fibrosis. Gastroenterology. 2020;159:1290301.e5. 
7 Panunzi S, De Gaetano A, Carnicelli A, Mingrone G. Predictors of remission of diabetes mellitus in severely obese individuals undergoing bariatric surgery: do BMI or procedure choice matter? A meta-analysis. Ann Surg. 2015;261:459-67.

8 Rubino F, Nathan DM, Eckel RH, Schauer PR, Alberti KG, Zimmet PZ, et al. Metabolic surgery in the treatment algorithm for type $2 \mathrm{di}$ abetes: a joint statement by international diabetes organizations. Surg Obes Relat Dis. 2016;12:1144-62.

9 Aminian A, Zajichek A, Tu C, Wolski KE, Brethauer SA, Schauer PR, et al. How much weight loss is required for cardiovascular benefits? Insights from a metabolic surgery matched-cohort study. Ann Surg. 2020;272: 639-45.

10 DGAV.S3-Leitlinie: Chirurgie der Adipositas und metabolischer Erkrankungen. AWMF online. 2018.

11 Das SR, Everett BM, Birtcher K, Brown JM, Januzzi JL Jr, Kalyani RR, et al. 2020 expert consensus decision pathway on novel therapies for cardiovascular risk reduction in patients with type 2 diabetes: a report of the American College of Cardiology Solution Set Oversight Committee. J Am Coll Cardiol. 2020;76:1117-45

12 Sjöström L. Bariatric surgery and reduction in morbidity and mortality: experiences from the SOS study. Int J Obes. 2008;32(Suppl 7): S93-7.

13 Sjöström L. Review of the key results from the Swedish Obese Subjects (SOS) trial: a prospective controlled intervention study of bariatric surgery. J Intern Med. 2013;273:219-34.

14 Mingrone G, Panunzi S, De Gaetano A, Guidone C, Iaconelli A, Capristo E, et al. Metabolic surgery versus conventional medical therapy in patients with type 2 diabetes: 10 year follow-up of an open-label, single-centre, randomised controlled trial. Lancet. 2021; 397:293-304.

15 Aminian A, Vidal J, Salminen P, Still CD, Nor Hanipah Z, Sharma G, et al. Late relapse of diabetes after bariatric surgery: not rare, but not a failure. Diabetes Care. 2020;43:534-40.

16 Syn NL, Cummins DE, Wang LZ, Lin DJ, Zhao JJ, Loh M, et al. Association of metabolic-bariatric surgery with long-term survival in adults with and without diabetes: a one-stage meta-analysis of matched cohort and prospective controlled studies with 174772 participants. Lancet. 2021;397:1830-41.

17 Müller-Stich BP, Fischer L, Kenngott HG, Gondan M, Senft J, Clemens G, et al. Gastric bypass leads to improvement of diabetic neuropathy independent of glucose normalization: results of a prospective cohort study (DiaSurg 1 study). Ann Surg. 2013;258:760-6.

18 Billeter AT, Scheurlen KM, Probst P, Eichel S, Nickel F, Kopf S, et al. Meta-analysis of metabolic surgery versus medical treatment for microvascular complications in patients with type 2 diabetes mellitus. Br J Surg. 2018;105:168-81.

19 Aminian A, Zajichek A, Arterburn DE, Wolski KE, Brethauer SA, Schauer PR, et al. Association of metabolic surgery with major adverse cardiovascular outcomes in patients with type 2 diabetes and obesity. JAMA. 2019; 322:1271-82.
20 Scheurlen KM, Probst P, Kopf S, Nawroth PP, Billeter AT, Müller-Stich BP. Metabolic surgery improves renal injury independent of weight loss: a meta-analysis. Surg Obes Relat Dis. 2019;15:1006-20.

21 Billeter AT, Kopf S, Zeier M, Scheurlen K, Fischer L, Schulte TM, et al. Renal function in type 2 diabetes following gastric bypass. Dtsch Arztebl Int. 2016;113:827-33.

22 Shulman A, Peltonen M, Sjöström CD, Andersson-Assarsson JC, Taube M, Sjöholm K, et al. Incidence of end-stage renal disease following bariatric surgery in the Swedish Obese Subjects Study. Int J Obes. 2018;42:964-73.

23 Cohen RV, Pereira TV, Aboud CM, Petry TBZ, Lopes Correa JL, Schiavon CA, et al. Effect of gastric bypass vs best medical treatment on early-stage chronic kidney disease in patients with type 2 diabetes and obesity: a randomized clinical trial. JAMA Surg. 2020; 55:e200420.

24 Younes R, Bugianesi E. NASH in lean individuals. Semin Liver Dis. 2019 Feb;39:86-95.

25 Fouad Y, Waked I, Bollipo S, Gomaa A, Ajlouni Y, Attia D. What's in a name? Renaming "NAFLD" to "MAFLD". Liver Int. 2020; 40:1254-61.

26 Piscaglia F, Svegliati-Baroni G, Barchetti A, Pecorelli A, Marinelli S, Tiribelli C, et al. Clinical patterns of hepatocellular carcinoma in nonalcoholic fatty liver disease: a multicenter prospective study. Hepatology. 2016;63:82738.

27 Lee Y, Doumouras AG, Yu J, Brar K, Banfield $\mathrm{L}, \mathrm{Gmora} \mathrm{S}$, et al. Complete resolution of nonalcoholic fatty liver disease after bariatric surgery: a systematic review and meta-analysis. Clin Gastroenterol Hepatol. 2019;17:104060.e11.

28 Kwak M, Mehaffey JH, Hawkins RB, Hsu A, Schirmer B, Hallowell PT. Bariatric surgery is associated with reduction in non-alcoholic steatohepatitis and hepatocellular carcinoma: a propensity matched analysis. Am J Surg. 2020;219:504-7.

29 Wirth KM, Sheka AC, Kizy S, Irey R, Benner A, Simon G, et al. Bariatric surgery is associated with decreased progression of nonalcoholic fatty liver disease to cirrhosis: a retrospective cohort analysis. Ann Surg. 2020;272: 32-9.

30 Rustgi VK, Li Y, Gupta K, Minacapelli CD, Bhurwal A, Catalano C, et al. Bariatric surgery reduces cancer risk in adults with nonalcoholic fatty liver disease and severe obesity. Gastroenterology. 2021;161(1):171-84.

31 Alam S, Jahid Hasan M, Khan MAS, Alam M, Hasan N. Effect of weight reduction on histological activity and fibrosis of lean nonalcoholic steatohepatitis patient. J Transl Int Med. 2019;7:106-14.

32 Brock JM, Billeter A, Müller-Stich BP, Herth F. Obesity and the lung: what we know today. Respiration. 2020;99:1-11.

33 Kershaw EE, Flier JS. Adipose tissue as an endocrine organ. J Clin Endocrinol Metab. 2004;89:2548-56.

34 Wang F, Xiong X, Xu H, Huang H, Shi Y, Li $\mathrm{X}$, et al. The association between obstructive sleep apnea syndrome and metabolic syndrome: a confirmatory factor analysis. Sleep Breath. 2019;23:1011-9.
35 Santamaria F, Montella S, Greco L, Valerio G, Franzese A, Maniscalco M, et al. Obesity duration is associated to pulmonary function impairment in obese subjects. Obesity. 2011; 19:1623-8.

36 Kuvat N, Tanriverdi H, Armutcu F. The relationship between obstructive sleep apnea syndrome and obesity: a new perspective on the pathogenesis in terms of organ crosstalk. Clin Respir J. 2020;14:595-604.

37 Alsumali A, Al-Hawag A, Bairdain S, Eguale $\mathrm{T}$. The impact of bariatric surgery on pulmonary function: a meta-analysis. Surg Obes Relat Dis. 2018;14:225-36.

38 Peralta GP, Marcon A, Carsin AE, Abramson MJ, Accordini S, Amaral AF, et al. Body mass index and weight change are associated with adult lung function trajectories: the prospective ECRHS study. Thorax. 2020;75:313-20.

39 Peters U, Hernandez P, Dechman G, Ellsmere J, Maksym G. Early detection of changes in lung mechanics with oscillometry following bariatric surgery in severe obesity. Appl Physiol Nutr Metab. 2016;41:538-47.

40 Schauer PR, Bhatt DL, Kirwan JP, Wolski K, Aminian A, Brethauer SA, et al. Bariatric surgery versus intensive medical therapy for diabetes: 5-year outcomes. N Engl J Med. 2017; 376:641-51.

41 Ikramuddin S, Korner J, Lee WJ, Thomas AJ, Connett JE, Bantle JP, et al. Lifestyle intervention and medical management with vs without Roux-en-Y gastric bypass and control of hemoglobin A1c, LDL cholesterol, and systolic blood pres- sure at 5 years in the Diabetes Surgery Study. JAMA. 2018;319:266-78.

42 Schiavon CA, Bhatt D, Ikeoka D, Santucci EV, Santos RN, Damiani LP, et al. Three-year outcomes of bariatric surgery in patients with obesity and hypertension: a randomized clinical trial. Ann Intern Med. 2020;173(9):685-93.

43 Aminian A, Brethauer SA, Andalib A, Nowacki AS, Jiminet A, Corcelles R, et al. Individualized metabolic surgery score: procedure selection based on diabetes severity. Ann Surg. 2017;266(4):650-7.

44 Duarte-Guerra LS, Coêlho BM, Santo MA, Wang YP. Psychiatric disorders among obese patients seeking bariatric surgery: results of structured clinical interviews. Obes Surg. 2015;25:830-7.

45 Peterli R, Wölnerhanssen BK, Peters T, Vetter D, Kröll D, Borbély Y, et al. Effect of laparoscopic sleeve gastrectomy vs laparoscopic Roux-en-Y gastric bypass on weight loss in patients with morbid obesity: the SM-BOSS randomized clinical trial. JAMA. 2018;319:255-65.

46 Salminen P, Helmiö M, Ovaska J, Juuti A, Leivonen M, Peromaa-Haavisto P, et al. Effect of laparoscopic sleeve gastrectomy vs laparoscopic Roux-en-Y gastric bypass on weight loss at 5 years among patients with morbid obesity: the SLEEVEPASS randomized clinical trial. JAMA. 2018;319:241-54.

47 Robert M, Espalieu P, Pelascini E, Caiazzo R, Sterkers A, Khamphommala L, et al. Efficacy and safety of one anastomosis gastric bypass versus Roux-en-Y gastric bypass for obesity (YOMEGA): a multicentre, randomised, open-label, non-inferiority trial. Lancet. 2019 393:1299-309. Erratum in: Lancet. 2019; 393(10178):1298. 